\title{
Comment on Choi et al. Correlation between Ionospheric TEC and the DCB Stability of GNSS Receivers from 2014 to 2016. Remote Sens. 2019, 11, 2657
}

\author{
Jiahao Zhong ${ }^{1} \mathbb{D}$, Jiuhou Lei ${ }^{2,3,4, * \mathbb{C}}$ and Xinan Yue ${ }^{5,6}$ \\ 1 Planetary Environmental and Astrobiological Research Laboratory (PEARL), \\ School of Atmospheric Sciences, Sun Yat-Sen University, Zhuhai 519082, China; \\ zhongjh55@mail.sysu.edu.cn \\ 2 CAS Key Laboratory of Geospace Environment, School of Earth and Space Sciences, \\ University of Science and Technology of China, Hefei 230026, China \\ 3 Mengcheng National Geophysical Observatory, University of Science and Technology of China, \\ Hefei 230026, China \\ 4 CAS Center for Excellence in Comparative Planetology, Hefei 230026, China \\ 5 Key Laboratory of Earth and Planetary Physics, Institute of Geology and Geophysics, \\ Chinese Academy of Sciences, Beijing 100029, China; yuexinan@mail.iggcas.ac.cn \\ 6 Innovation Academy for Earth Science, Chinese Academy of Sciences, Beijing 100029, China \\ * Correspondence: leijh@ustc.edu.cn
}

Received: 19 June 2020; Accepted: 22 October 2020; Published: 24 October 2020

\begin{abstract}
Choi et al. (2019) analyzed the correlation between the ionospheric total electron content (TEC) and the Global Navigation Satellite System (GNSS) receiver differential code bias (DCB) and concluded that the long-term variations of the receiver DCB are caused by the corresponding variations in the ionosphere. Unfortunately, their method is problematic, resulting in conclusions that are not useful. The long-term variations of the Global Positioning System (GPS) DCBs are primarily attributed to the GPS satellite replacement with different satellite block series under the zero-mean constraint condition, rather than the ionospheric variability.
\end{abstract}

Keywords: GPS DCB; receiver DCB; DCB stability; ionospheric variability; satellite replacement

In the ionospheric total electron content (TEC) retrieval, the differential code bias (DCB) caused by the Global Navigation Satellite System (GNSS) satellite transmitter and receiver hardware is one of the main error sources, and it has been widely studied. Choi et al. [1] have recently proposed a new aspect of the correlation between the ionospheric TEC and the GNSS receiver differential code bias (rDCB). They stated that "the characteristics of rDCB and rDCB root mean square (RMS) have a similarity with those of vertical TEC (VTEC), at all stations", and they calculated Pearson's coefficient (R) between the TEC and rDCB and showed that "the correlation between TEC and rDCB is the smallest in low-latitude regions. The mid-latitude regions exhibit the maximum value of R". Supposedly, the GNSS DCB should be related with the transmitter hardware and its environment, as expected. Studies also showed that the variations of the Global Positioning System (GPS) satellite DCB and receiver DCB are not caused by the ionospheric variability [2,3]. However, Choi et al. [1] might have advertently or inadvertently overlooked the work of Zhong et al. [2,3]. As discussed subsequently, it does not make sense to utilize the comparison between the ionospheric TEC and the GNSS receiver DCB to demonstrate their cause-effect relationship. Thus, the correlation analysis between TEC and rDCB as described by Choi et al. [1] is not meaningful. 
Zhong et al. [2,3] have assessed the variations of the GPS satellite DCB and receiver DCB. Note that in the DCB estimation, only the combined satellite-receiver DCB (the sum of the GPS DCB and receiver DCB) can actually be determined, so that an additional constraint condition is required to further separate the GPS DCB and receiver DCB. Generally, the zero-mean condition imposed on all satellite DCBs (i.e., the daily mean of all GPS DCBs is set as zero) is introduced for the DCB separation [4]. Figure 1 shows the comparisons of the GPS DCB of Pseudo Random Number (PRN) 13 from the International GNSS Service (IGS) [5] and solar activity proxy F10.7 during 1998-2020. It seems that the GPS DCB also showed a solar-cycle-like variation [6], if only the period of 2002-2014 is considered. However, this is not the case for the whole period of 1998-2020.

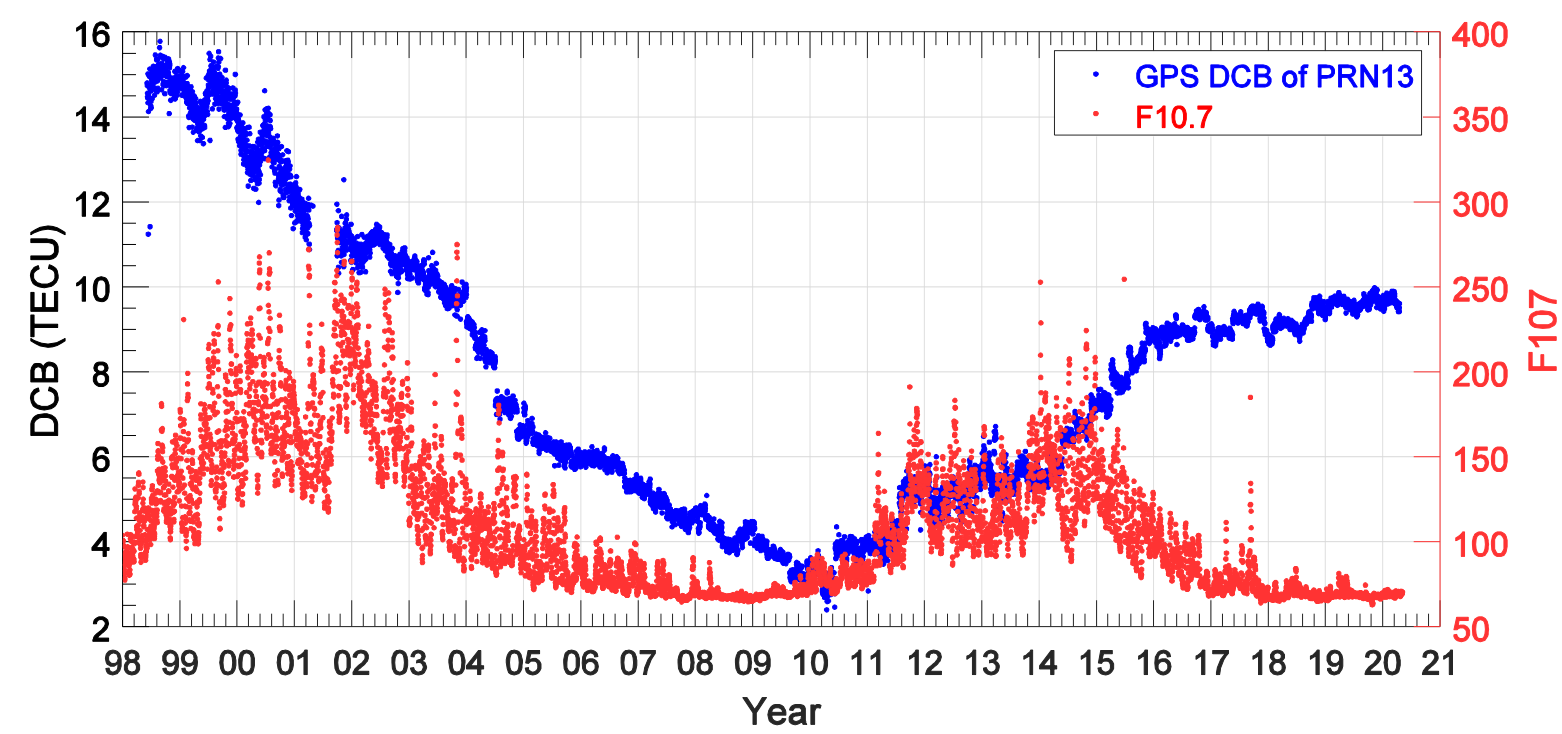

Figure 1. Variations of the International Global Navigation Satellite System (GNSS) Service (IGS) Global Positioning System (GPS) differential code bias (DCB) of Pseudo Random Number (PRN) 13 and solar activity proxy F10.7 from 1998 to 2020.

Figure 2 presents the lifetime of each GPS satellite from 1998 to 2020 . It is clear that the new satellite block series are replacing the old ones gradually. From the GPS DCB values on 1 January 2015 as displayed at the upper left corner of Figure 2, it is evident that the GPS DCBs from different satellite block series show a significant difference. Under the zero-mean condition imposed on all GPS DCBs, when a new GPS satellite replaces a decommissioned one with a different block series, the GPS DCB values change relatively to satisfy the zero-mean condition. Specifically, when a new GPS satellite with higher (lower) DCB values comes into service, all the GPS DCB values descend (increase). Thus, the long-term variations of the GPS DCBs are not caused by the ionospheric variability, while they are attributed to the GPS satellite replacement with different satellite block series under the zero-mean condition [2]. Correspondingly, the estimated receiver DCB will also change, as the daily combined satellite-receiver DCB is constant. Note that only after the effect of GPS satellite replacement is removed, the DCB could be used for comparison or periodic spectral analysis.

To remove the effect of GPS satellite replacement, we can reconstruct the DCB value under a new constraint condition imposed only on continuously operating GPS satellites during a specific period. The new constraint condition for the GPS DCBs is expressed as follows: 


$$
\frac{1}{n} \sum_{i=1}^{n} D C B_{\mathrm{s}, \mathrm{rec}}^{i}=\mathrm{C}
$$

where $D C B_{\mathrm{s}, \text { rec }}$ denotes the reconstructed GPS DCB, $n$ is the number of the selected GPS satellites, and $C$ is a constant value. During the period of 2005-2019, nine continuously operating GPS satellites (PRN $11,13,14,16,19,20,22,23$, and 28) are selected. For each day, the offset value is calculated based on the mean value of the GPS DCBs of the selected satellites:

$$
D C B_{\text {offset }}=\frac{1}{n} \sum_{i=1}^{n} D C B_{\mathrm{s}}^{i}-\mathrm{C}
$$

Then, the reconstructed GPS DCB $\left(D C B_{\mathrm{s}, \text { rec }}\right)$ can be obtained from the original GPS DCB and the offset value as follows:

$$
D C B_{\mathrm{s}, \mathrm{rec}}=D C B_{s}-D C B_{\text {offset }}
$$

The receiver $\mathrm{DCB}\left(D C B_{\mathrm{r}, \mathrm{rec}}\right)$ can be also obtained in the similar way, but with an opposite sign on the offset value:

$$
D C B_{\mathrm{r}, \text { rec }}=D C B_{r}+D C B_{\text {offset }}
$$

The reconstructed GPS DCB of PRN28 is shown in Figure 3a as an example. On the long-term scale, the reconstructed GPS DCB only showed a slight variation of less than 1 TECU. In addition, the reconstructed GPS DCB presented short-term periodic variations, which is less than one year. The Lomb-Scargle periodograms of the original and reconstructed GPS DCBs are shown in Figure $3 \mathrm{~b}$. It is clear that the original GPS DCB presented multiple periodic components, while some periodic components in the reconstructed GPS DCB became much weaker. This indicates that DCB reconstruction could filter out the artificial periodic variations. In addition, it is interesting that there is a prominent periodic component of about 175 days in Figure 3b, which is a little shorter than half of a sidereal year (182.625 days). Zhong et al. [3] demonstrated that the periodic variation of CHAMP satellite receiver DCB is mainly attributed to its hardware thermal status. Here, we calculated the daily mean solar angle on the zenith side of the GPS satellite to represent its hardware thermal status, as shown in Figure 3c. The periodogram of the daily mean solar angle in Figure $3 \mathrm{~d}$ displayed a clear periodic component of about 175 days, which is generally in agreement with that of the GPS DCB. This period is matched with the period of a GPS draconitic year, which is equal to about 351.5 days. The GPS draconitic year is the time taken for the Sun to complete one revolution (return to the same point in space) with respect to the GPS orbital nodes, as seen from the Earth $[7,8]$. In one GPS draconitic year, there are exactly two eclipsing periods, which means that after 175.75 days, the elevation of the Sun above the orbital plane is the same, and the direction of the relative change in the height of the Sun is also the same. As a result of the Sun illumination and attitude of GPS satellites [9], every 175.75 days the Sun illuminates the same parts (electron circuits) of the GPS satellite in the same manner, which may change the hardware thermal status with the same period and further affect the DCB values. The periodic component of about 175 days can be seen in DCB around half of the total GPS satellites (not shown), but it is still not clear whether the effect of hardware thermal status on DCB is consistent for different GPS satellite block series. Many other GNSS-derived parameters are characterized by the draconitic repeatabilities or their harmonics, such as GNSS-based Earth Rotation Parameters [10]. Overall, both the solar-cycle-like and semiannual-like variations of the GPS DCB are artificial and not caused by the ionospheric variability. 


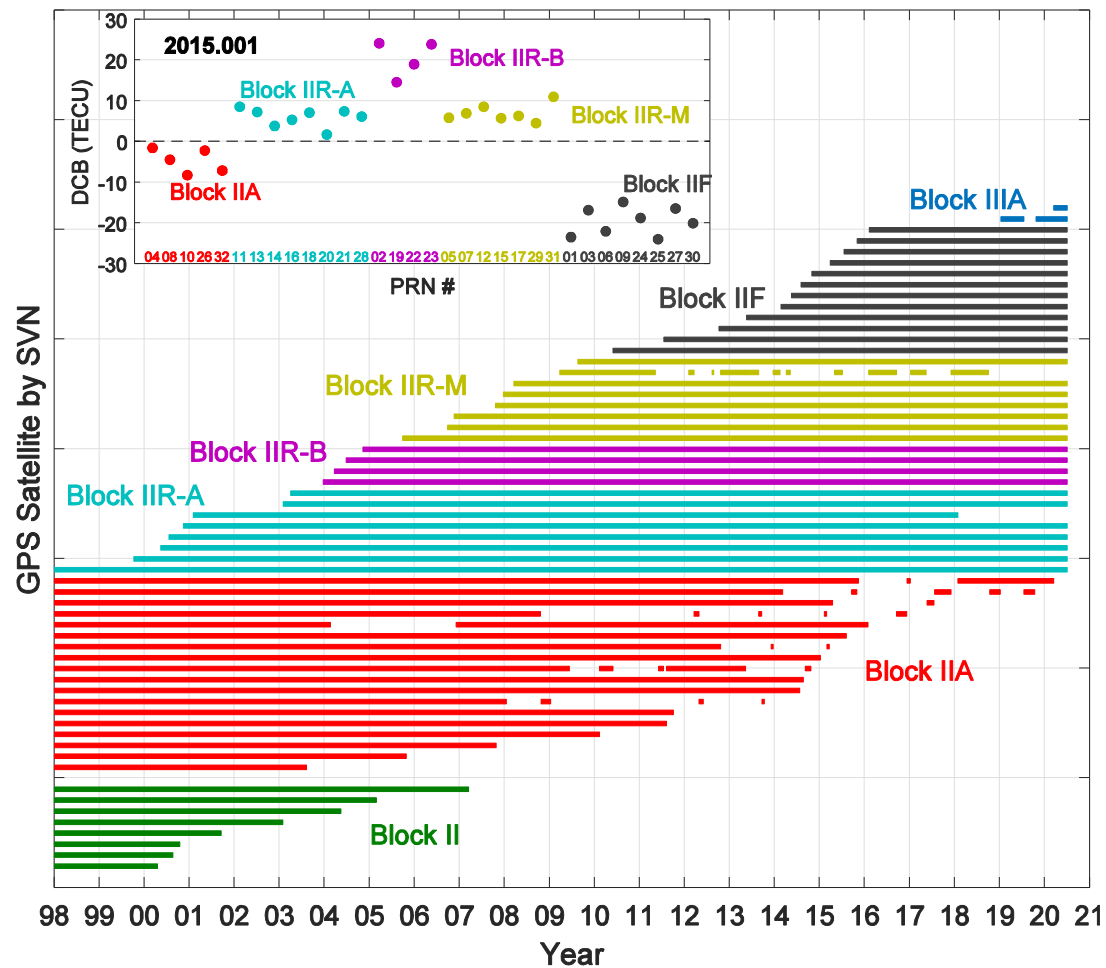

Figure 2. Lifetime of each GPS satellite sorted by Space Vehicle Number (SVN) from 1998 to 2020. Different colors denote different satellite block series. The GPS DCBs on 1 January 2015 are displayed on the upper left corner in the figure, with the corresponding PRNs shown at the bottom.
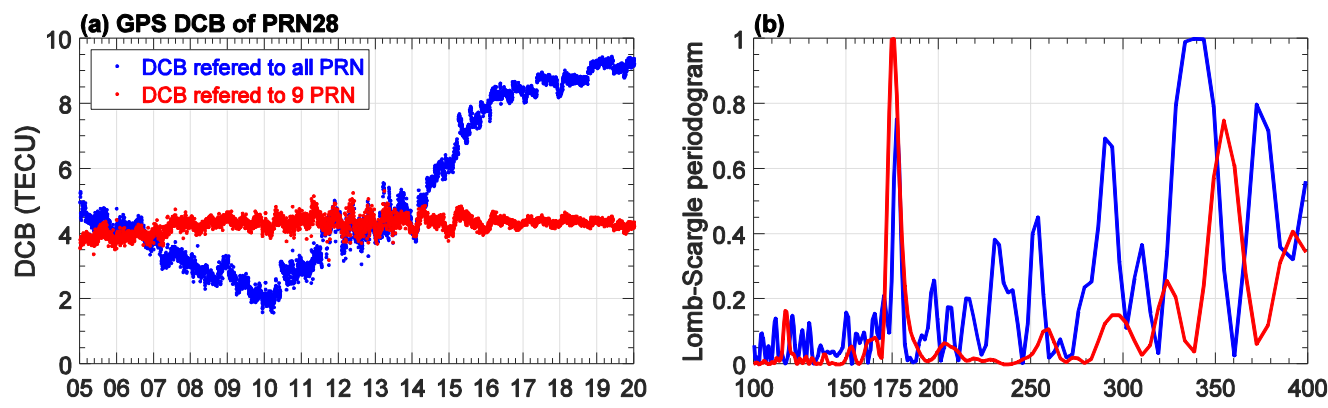

50 (c) Daily mean solar angle on zenith side of PRN28
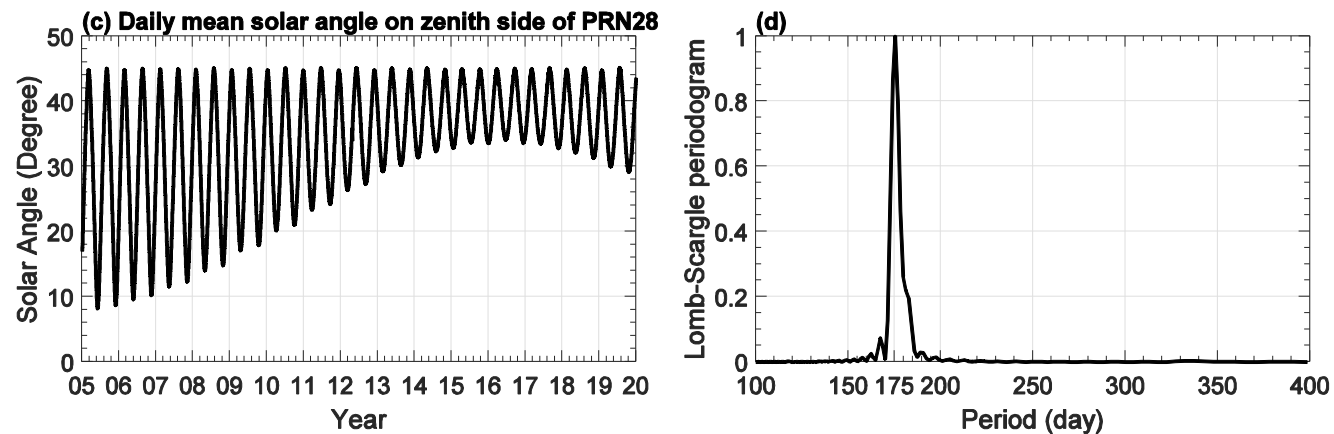

Figure 3. Variations of (a) GPS DCB and (c) daily mean solar angle on the zenith side of PRN28 (SVN44, Block IIR-A) from 2005 to 2019. The Lomb-Scargle periodograms of GPS DCB and daily mean solar angle are shown on the right panels in $(\mathbf{b}, \mathbf{d})$, respectively. In $(\mathbf{a}, \mathbf{b})$, the blue dots denote the original GPS DCBs from IGS, and the red dots denote the reconstructed GPS DCBs under the constraint condition imposed on the GPS DCBs of 9 continuously operating satellites. In $(\mathbf{b}, \mathbf{d})$, the power spectral density is scaled by the maximum value within the interest periods. 
Figure 4 shows the original and reconstructed receiver DCBs for station GRAS as an example, as used in Choi et al. [1]. Again, the original receiver DCB presented a declined trend from 2014 to 2016 , when F10.7 was mainly decreasing. However, the reconstructed receiver DCB only changed slightly during 2012-2017. The periodograms showed that the receiver DCB also presents a significant periodic component of about 176 days, which should be associated with the GPS draconitic year, as discussed above. In addition, there is a significant annual periodic component (365 days). The short-term variations of the receiver DCB are attributed to various factors, such as the hardware temperature [11]. From the comparison between surface temperature and the reconstructed receiver DCBs from many IGS stations (not shown), it illustrated that when the surface temperature reached its maximum (minimum), some of the reconstructed receiver DCBs also presented the maximum (minimum) values. Thus, the annual periodic component could probably be associated with the variations of the surface temperature. However, the manufacturer and model, antenna mounting position, cable construction, and thermal control could be complicated and different for each receiver, so it is difficult to draw a unified conclusion in respect of short-term variations of the receiver DCB.
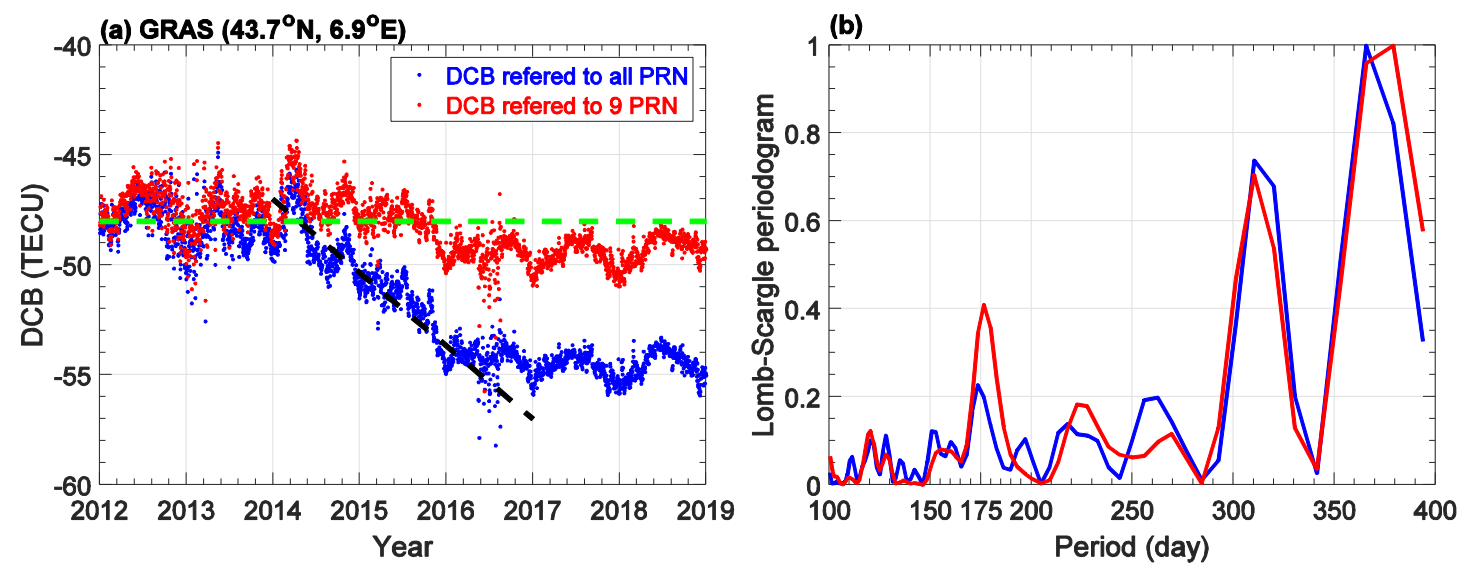

Figure 4. The same as Figure 3a,b, but for the (a) receiver DCBs at GRAS station, and (b) its Lomb-Scargle periodograms during 2012-2018. The green and black dashed lines indicate the variation trend of the DCBs for reference.

As discussed above, both the long-term and short-term variations of the GPS DCBs and receiver DCBs are accessed. Though during specific periods, they were similar to the ionospheric variations by coincidence, they are not caused by the ionospheric variability physically. In the ionospheric TEC/DCB estimation, it is generally assumed that the ionosphere satisfies the spherically symmetric hypothesis or spherical harmonic expansion distribution. When the actual ionospheric distributions deviate from the assumption, the TEC measurement used in the DCB determination is subject to a large number of small random errors, and the probability distribution for the total statistical error is driven toward the Gaussian distribution, indicated by higher RMS (lower stability) for DCB. In TEC/DCB estimation, the larger the ionospheric TEC is, the greater the deviation from the spherically symmetric assumption would be, thus causing higher RMS (lower stability) for DCB, as presented by Choi et al. [1]. However, as mentioned above, in the physical sense the ionospheric TEC cannot alter the rDCB, which is essentially subject to the receiver hardware.

In summary, Choi et al. [1] concluded arbitrarily that the long-term variations of the receiver DCB are caused by the ionospheric variability on the basis of the problematic method. They were not aware of the effect of the GPS satellite replacement on the DCB determination. Overall, the study of Choi et al. [1] could introduce confusion and be misleading for the GNSS and the ionospheric communities. 
Author Contributions: J.L. designed the experiment to evaluate the DCB variations. J.Z. conducted the experiment and prepared the first draft of the comment. J.L. and X.Y. intensively contributed to the text and the discussion. All authors have read and agreed to the published version of the manuscript.

Funding: This work was supported by the B-type Strategic Priority Program of the Chinese Academy of Sciences (XDB41000000), the National Natural Science Foundation of China (41831070, 41974181), the Open Research Project of Large Research Infrastructures of CAS - "Study on the interaction between low/mid-latitude atmosphere and ionosphere based on the Chinese Meridian Project". J. Z. was also supported by the National Natural Science Foundation of China (41804150).

Acknowledgments: We thank the IGS for the GPS DCB and receiver DCB data (ftp://igs.ensg.ign.fr/pub/ igs/products/ionosphere/) and thank CODE for the specific GPS satellite information (ftp://ftp.aiub.unibe.ch/ BSWUSER52/GEN/SATELLIT.I14).

Conflicts of Interest: The authors declare no conflict of interest.

\section{References}

1. Choi, B.-K.; Sohn, D.-H.; Lee, S.J. Correlation between Ionospheric TEC and the DCB Stability of GNSS Receivers from 2014 to 2016. Remote Sens. 2019, 11, 2657. [CrossRef]

2. Zhong, J.; Lei, J.; Dou, X.; Yue, X. Is the long-term variation of the estimated GPS differential code biases associated with ionospheric variability? GPS Solut. 2016, 20, 313-319. [CrossRef]

3. Zhong, J.; Lei, J.; Yue, X.; Dou, X. Determination of differential code bias of GNSS receiver onboard low Earth orbit satellite. IEEE Trans. Geosci. Remote Sens. 2016, 54, 4896-4905. [CrossRef]

4. Schaer, S. Mapping and Predicting the Earth's ionosphere Using the Global Positioning System. Ph.D. Thesis, University of Bern, Bern, Switzerland, 1999.

5. Hernández-Pajares, M.; Juan, J.M.; Sanz, J.; Orus, R.; Garcia-Rigo, A.; Feltens, J.; Komjathy, A.; Schaer, S.C.; Krankowski, A. The IGS VTEC maps: A reliable source of ionospheric information since 1998. J. Geod. 2009, 83, 263-275. [CrossRef]

6. Zhang, D.H.; Shi, H.; Jin, Y.Q.; Zhang, W.; Hao, Y.Q.; Xiao, Z. The variation of the estimated GPS instrumental bias and its possible connection with ionospheric variability. Sci. China Tech. Sci. 2014, 57, 67-79. [CrossRef]

7. Ray, J.; Altamimi, Z.; Collilieux, X.; van Dam, T. Anomalous harmonics in the spectra of GPS position estimates. GPS Solut. 2008, 12, 55-64. [CrossRef]

8. Allahverdi-zadeh, A.; Asgari, J.; Amiri-Simkooei, A.R. Investigation of GPS draconitic year effect on GPS time series of eliminated eclipsing GPS satellite data. J. Geod. Sci. 2016, 6, 93-102. [CrossRef]

9. Montenbruck, O.; Schmid, R.; Mercier, F.; Steigenberger, P.; Noll, C.; Fatkulin, R.; Kogure, S.; Ganeshan, A.S. GNSS satellite geometry and attitude models. Adv. Space Res. 2015, 56, 1015-1029. [CrossRef]

10. Zajdel, R.; Sośnica, K.; Bury, G.; Dach, R.; Prange, L. System-specific systematic errors in earth rotation parameters derived from GPS, GLONASS, and Galileo. GPS Solut. 2020, 24, 74. [CrossRef]

11. Coster, A.; Williams, J.; Weatherwax, A.; Rideout, W.; Herne, D. Accuracy of GPS total electron content: GPS receiver bias temperature dependence. Radio Sci. 2013, 48, 190-196. [CrossRef]

Publisher's Note: MDPI stays neutral with regard to jurisdictional claims in published maps and institutional affiliations.

(C) 2020 by the authors. Licensee MDPI, Basel, Switzerland. This article is an open access article distributed under the terms and conditions of the Creative Commons Attribution (CC BY) license (http://creativecommons.org/licenses/by/4.0/). 